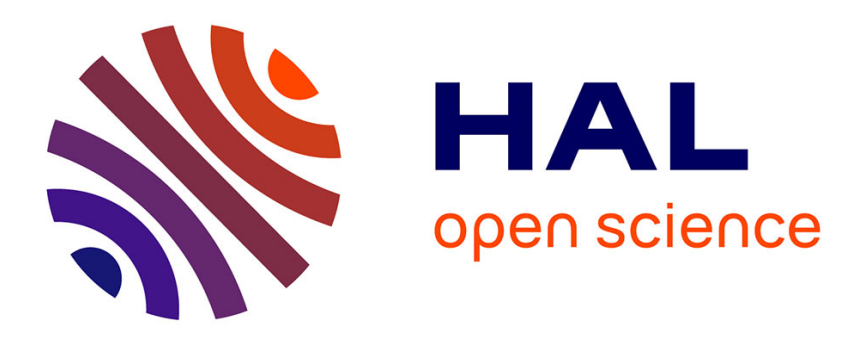

\title{
Jonathan Gabe, Lee F. Monaghan, Key Concepts in Medical Sociology
}

Maël Dieudonné

\section{To cite this version:}

Maël Dieudonné. Jonathan Gabe, Lee F. Monaghan, Key Concepts in Medical Sociology. 2013, http://lectures.revues.org/12040. halshs-00973378

\section{HAL Id: halshs-00973378 \\ https://shs.hal.science/halshs-00973378}

Submitted on 8 Apr 2014

HAL is a multi-disciplinary open access archive for the deposit and dissemination of scientific research documents, whether they are published or not. The documents may come from teaching and research institutions in France or abroad, or from public or private research centers.
L'archive ouverte pluridisciplinaire HAL, est destinée au dépôt et à la diffusion de documents scientifiques de niveau recherche, publiés ou non, émanant des établissements d'enseignement et de recherche français ou étrangers, des laboratoires publics ou privés. 


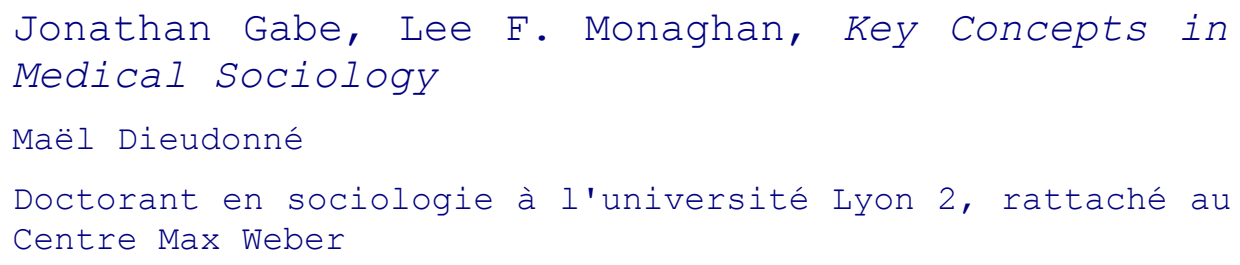

L'ouvrage recensé ici est la seconde édition remaniée d'un manuel originellement publié en 2004, et dont le succès a été immédiat (il a bénéficié de huit réimpressions jusqu'en 2011). Selon le principe de la collection à laquelle il appartient, ses auteurs ont adopté une entrée par les concepts, à laquelle ils entendent cependant donner une tournure problématisée et critique : il s'agit moins pour eux de proposer un dictionnaire, que de donner à voir le champ de la sociologie médicale dans son ensemble, à travers les grandes notions qui ont contribué à le façonner et l'orientent aujourd'hui. Ce manuel est en effet destiné aux étudiants de licence, et sa réédition est justifiée autant par l'évolution de ses objets (découlant par exemple de l'accroissement du rôle d'Internet et du renforcement de la sensibilité au risque médical) que par les progrès de leur connaissance sociologique. Il présente 49 contributions réparties en cinq chapitres.

Le premier est consacré au modelage («patterning ») social de la santé : il s'attache à montrer comment certaines divisions sociales se répercutent sur la santé des individus, ou plus exactement influencent les variables permettant de l'appréhender (essentiellement les taux de mortalité et de morbidité). Sont ainsi étudiés les effets de l'appartenance sociale, du genre, de l'ethnicité et de l'âge, mais aussi ceux du lieu de résidence et des perturbations («disruptions») dans les trajectoires biographiques.

Le second chapitre traite de la santé et de la maladie comme expériences vécues, ainsi que des rapports entre les malades et les professionnels du soin : ses contributions s'appuient largement sur les traditions nord-américaines du fonctionnalisme et de l'interactionnisme, qui analysent la maladie comme une forme de déviance. Y sont évidemment abordés les notions de stigmate, de handicap et de maladie chronique, de rôle de malade et de récit de maladie ("illness narrative »), mais aussi des thèmes plus généraux comme la médicalisation, le risque ou la qualité de vie.

Le troisième chapitre porte sur la connaissance médicale et les pratiques thérapeutiques. Il rassemble des contributions relatives à la biomédecine et aux multiples techniques sur lesquelles elle s'appuie désormais, aux savoirs profanes et à leur tendance à la généticisation, aux débats suscités par et consacrés à la bioéthique, à la santé en ligne ( eHealth»), ou encore à la « médecine de surveillance » cherchant à améliorer la santé globale de la population en s'inspirant d'observations épidémiologiques.

Le quatrième chapitre déplace le regard vers les professionnels du soin et la division du travail médical. Y sont notamment étudiés la domination des médecins et son revers : la confiance que les patients leur accordent, la professionnalisation de 
certains métiers de la santé (à travers les exemples des infirmières et des sagesfemmes) et le travail émotionnel qu'ils impliquent, les soins informels et les médecines alternatives, ou encore les migrations internationales des praticiens.

Dans le dernier chapitre est adoptée une perspective plus globale, puisqu'il est consacré aux institutions du système de santé et aux politiques sanitaires. On y rencontre une contribution sur l'hôpital, une autre sur la régulation du marché des médicaments, et quelques autres encore sur les grands totems des réformes implémentées au Royaume-Uni et en Europe ces dernières années : privatisation, gestion managériale, orientation client («consumerism »), évaluation devant aboutir à l'instauration d'un « evidence-based health care »...

Ces contributions sont remarquablement homogènes tant par leur longueur (environ quatre pages) que leur structure : elles débutent par un encadré proposant une définition simple du concept étudié, dont elles précisent ensuite l'origine, puis recensent les principaux avatars dans la littérature sociologique. Elles s'achèvent par une conclusion synthétique ou prospective, doublée d'une bibliographie indicative comprenant une dizaine de références. Leur qualité générale est très satisfaisante. Presque toutes sont de bonne facture et réellement intéressantes ; une douzaine nous ont semblé vraiment excellentes, tandis que seulement six ou sept se sont avérées décevantes.

Le panorama qu'elles dessinent est relativement complet: l'arbitrage entre le nombre de concepts présentés et la précision de leur analyse est sans doute le plus délicat qu'impose l'élaboration de ce genre de manuel, mais l'équilibre retenu par les auteurs est convaincant. Il va sans dire que le choix d'aborder par les concepts un champ de recherche aussi vaste que celui de la sociologie médicale ne permet pas d'en donner une vision aussi systématique et structurée qu'un manuel bien problématisé, mais celui-ci, par les nombreux éclairages qu'il propose, tire beaucoup de ses recoins de l'ombre - il autorisera ainsi le lecteur français à (re)découvrir certains concepts de la sociologie anglophone peu diffusés dans la littérature nationale. Il en paye le prix par quelques redites et de petites incohérences, qu'il vaut mieux considérer comme des conséquences de sa fidélité : les qualités de cet ouvrage sont réelles, et devraient lui permettre de s'adresser à un public plus large que celui des étudiants auxquels il se destine.

À signaler enfin une introduction courte mais bien faite, proposant une histoire de la sociologie médicale aux États-Unis et au Royaume-Uni qui semble assez différente de ce qu'elle a été en France : dans les premiers elle s'est développée au sein des facultés de médecine et a d'abord été très orientée vers la pratique (c'est la fameuse «sociology in medicine» distinguée par Robert Straus en 1957). Elle s'est progressivement tournée vers le versant théorique, mais aussi pour l'ériger en source de légitimité. En France au contraire, la sociologie médicale s'est développée tardivement, en conjonction avec un discours critique de la domination médicale, et ne s'est institutionnalisée qu'après coup ${ }^{1}$.

\footnotetext{
${ }^{1}$ Herzlich Claudine, Pierret Janine, «Au croisement de plusieurs mondes : la constitution de la sociologie de la santé en France (1950-1985)», Revue Française de Sociologie, vol. 51(1), 2010, pp. 121-148
} 